\title{
The flexible perforated screens are a protection of buildings against external explosions
}

\author{
Vyacheslav Gorev ${ }^{1, *}$ and Grigoriy Medvedev ${ }^{2}$ \\ ${ }^{1}$ Moscow State University of Civil Engineering, Yaroslavskoe shosse, 26, Moscow, 129337, Russia \\ ${ }^{2}$ Agency of fire audit, Mozhayskoye highway, 29, st.1, Moscow, 121471, Russia
}

\begin{abstract}
This work studies the parameters of the blast wave passing through a screen placed in its path. It determines the degree of pressure reduction behind the barrier depending on its permeability, the relative size of the obstacles, the length of the positive phase of the wave, the degree of removal of waves from the source. It also determines the size of the zone of decompression. Experiments are made with models of buildings that are located behind a barrier in the course of wave motion. These experiments gave grounds to conclude that such obstacles can serve as an effective explosion protection. It is proposed to use a flexible sheet as elements subject to blast load. It is shown that a sheet of soft steel, with a thickness of $1 \mathrm{~mm}$ and a length of $6000 \mathrm{~mm}$ can take a maximum load of $100 \mathrm{kPa}$. The strengthening of the sheet by increasing its effective thickness in proportion to extends its capabilities.
\end{abstract}

\section{Introduction}

Emergency explosions of vapour clouds in open space (VCE) occur regularly and cause considerable material damage and human losses [1-5]. Despite that, explosions occur in the open space, the vast number of casualties found inside of buildings when they collapse from the impact of the explosion [4,6]. But the wave itself does not cause deaths in open space. Destructible buildings and the associated falls of heavy structures lead to fatal outcomes. We are talking about $\mathrm{VCE}$, when the maximum excess pressure in the wave rarely exceeds a value of $\Delta \mathrm{P}=100 \mathrm{kPa}$. It is necessary to provide blast-proof buildings with permanent stay of people at enterprises where VCE is possible. They are administrative buildings, laboratories and control points $[7,20]$.

The investigation of the explosion in Fliksborough in UK [6] showed that the staff that were on the street were not injured. But people that were in the buildings that were destroyed, were killed. VCE also destroy buildings, located in the nearby settlements. Fliksborough in explosion destroyed 1821 of buildings, including 167 shops and other offices located outside the plant. The explosion in Mexican city San Juan destroyed 100 houses and 200000 people were evacuated [4].

Explosions on trunk pipelines and product pipelines impact the nearby communities. The explosion on the product pipeline (Ufa, June 4, 1984.) destroyed buildings at a distance

*Corresponding author: ICA_kbs@mgsu.ru 
of $7 \mathrm{~km}$ from the epicenter [3]. Schools, supermarkets, theaters, large stadiums, and other structures with the presence of a large number of people could also be destroyed. The explosion-resistance of objects could be achieved:

- at the design stage, taking into account possible explosive effects;

- under reconstruction and operation if the conditions of explosion safety of the object has changed.

In the first case, the necessary margin of safety of bearing structures is required. In the second case, the shields located between the sources of the explosion and the object could be used. The protective barrier is located close to the object before him during VCE. In this case the protected object is covered by an area of low pressure (shadow).

Interest in VCE periodically increased due to obtaining new results on turbulent combustion [8-11], the initiation of various modes of blast, the parameters of blast waves in VCE $[5,12,21]$ on the nature of failure at these explosions. Thus, currently, there is a possibility to develop a method of protection from external explosion of sites that require this protection. At the moment the construction of solid barriers in front of the object is the most simple and the only existing method of protection.

For now the barriers, load-bearing capacity which was larger than that of the protected object were designed.

In this paper we propose to use a perforated barrier which compared to the solid, less material and creates greater length of the zone of reduced pressure. In addition, in the present work for the first time proposes to use as a protective element in a flexible sheet of mild steel. Such a sheet can withstand heavy loads without losing integrity due to deep plastic deformations.

\section{Materials and Methods}

The effect of barriers on the distribution of hemispherical blast wave.

In this work we study the possibility of protecting the facility from external explosion with a screen in front of him from a potential source of explosion. The action of such a screen is illustrated in Figure 1.

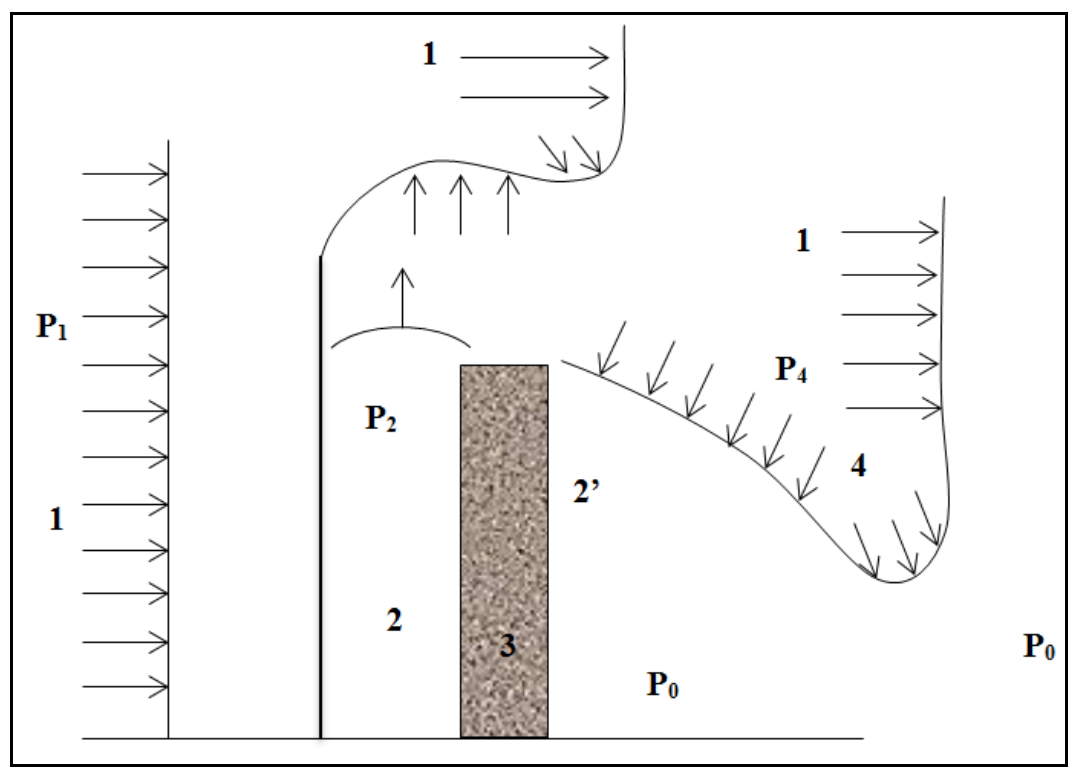

Fig. 1. The action of such a screen. 
Zone 1 - incident wave, marked by the pressure $\mathrm{P}_{1}$. After the interaction the wave screen (3) is formed in the reflection area (2) marked with a pressure $\mathrm{P}_{2}>\mathrm{P}_{1}$. From zone (2) up will spread a wave of compression, which will shift the zone (1) up. After passing over the obstacle (3) area (1) (the incident wave) will descend in the undisturbed zone (0), marked by the pressure $\mathrm{P}_{0}$.

The result is a zone (4) (numb) wave pressure $\mathrm{P} 4$ that satisfy the condition $\mathrm{P}_{0}<\mathrm{P}_{4}<\mathrm{P}_{1}$.

Gradually, the area (4) replaces the zone (0) if the length of the incident wave is large enough, $\mathrm{C}_{0}$ is the speed of sound, $\mathrm{t}_{+}$- duration positive phase of the wave. Otherwise, the wave moves away from the barrier, not breaking zone (0). This happens if the barrier is solid.

In the case of a perforated barrier zone (2) penetrates through the barrier, forming a zone (2'). Area (2') makes it difficult the penetration zone (4) and therefore a low pressure zone in this case (2') has a greater length than in the case of solid obstacles.

Decrease of parameters of the blast waves behind a perforated screen, was investigated in shock tubes $[13,14]$. The experiments were conducted with an incident shock wave, the intensity of the feeding wave was varied from 1 to $200 \mathrm{kPa}$. The attenuation coefficient of pressure K1 (the ratio of the pressure behind barrier to the pressure of in front of obstacles), almost linearly increases from 0.03 to 0.9 at the change of permeability barriers $\Omega$ from 0.01 to 0.8 . The experiments were carried out in conditions when all of the gas passed through the barrier, i.e. the gaps between the pipe wall and the obstacle was absent. In [13] it is concluded that when $\Omega<0.2$ the reflection of the shock wave at the perforated barrier is almost like a solid barrier. In [15] gives an empirical relationship of attenuation of compression waves with an intensity of from 2 to $70 \mathrm{kPa}: \mathrm{K}_{1}=1.14 \Omega^{0.62}$.

Nothing has been said on the influence of obstacles on wave from deflagration explosion in free space do not exist till now.

This work spreads the light on this issue. The experimental setup described in [16-18]. In the first series of experiments as the barriers used flat solid screens, in which the ratio of height to width is $H / B=0.8$. Thus, the characteristic size of the obstacles is $l_{x}=\min (H, B / 2)$ its half-width. The obstacle is placed symmetrically about a line connecting the center of the explosion with her middle. The radius of the cloud of products of combustion in all experiments was $0.4 \mathrm{~m}$. The layout of pressure sensors is shown in Figure 2 . 


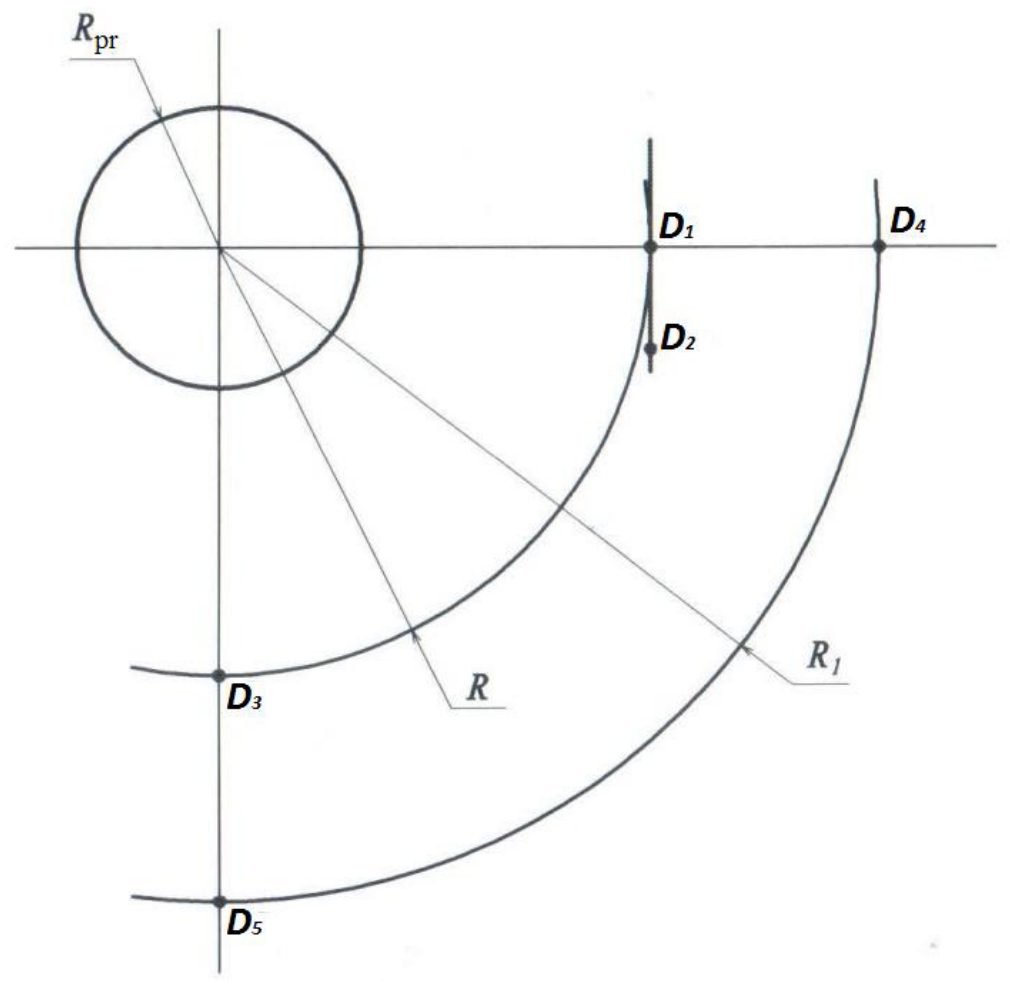

Fig. 2. Scheme of an arrangement of sensors.

To assess the impact of obstacles on the wave parameters used coefficient of attenuation of waves behind a barrier, $\mathrm{K}_{1}$, is equal to the ratio of the maximum excess pressure at the point behind the obstacle $\left(\mathrm{D}_{1}\right)$ to the maximum overpressure in a freely propagating wave at the same distance from the center of the explosion $\left(D_{3}\right)$. As a result of experiments it was determined: $\mathrm{K}_{1}$ does not depend on the values of maximum overpressure in the blast wave $\Delta \mathrm{P}_{\mathrm{m}}$, and is determined only by the ratio of the geometric characteristics of the: $l_{x} / R$, $\bar{\lambda}=\left(\tau_{+} C_{0}\right) / l_{x},\left(R_{1}-R\right) / l_{x}$. In Figure 3 and Figure 4 shows the dependence of $\mathrm{K}_{1}$ on the nature of the immersion of the object in the wave $\bar{\lambda}=C_{0} t_{+} / l_{x}$, where $\mathrm{t}_{+}$is the time of pressure rise at deflagrations the explosion and depending on the removal of the center of the explosion from the barrier $\mathrm{R}$. 


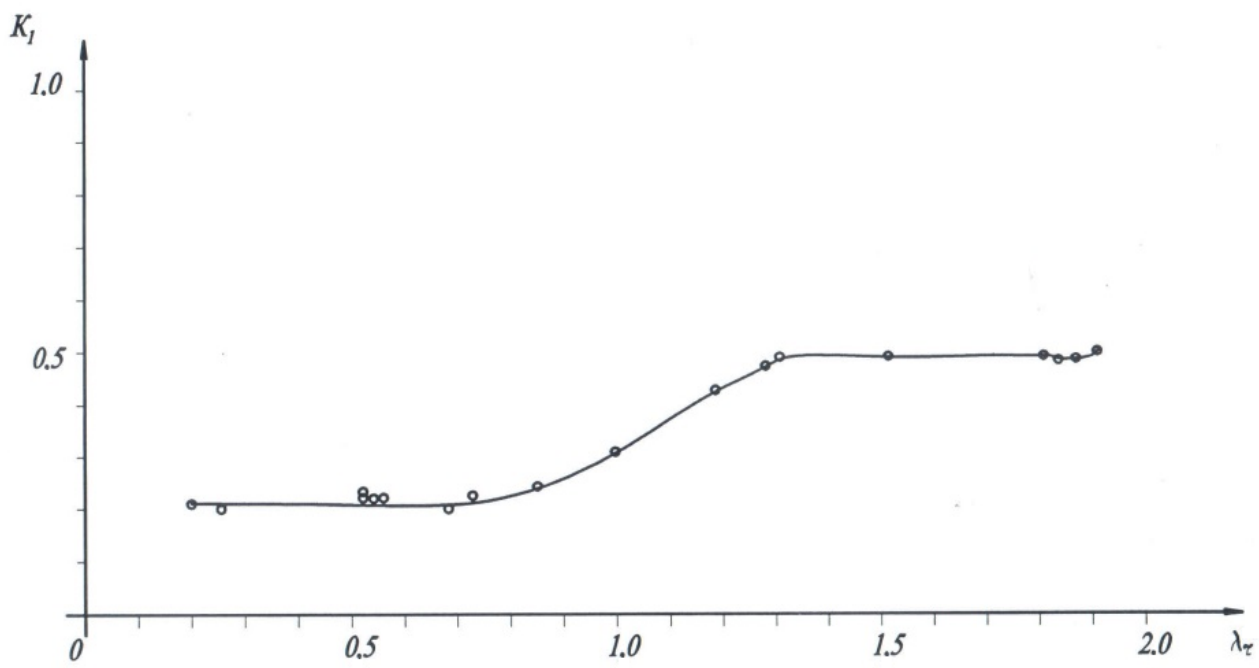

Fig. 3. Dependence of an extinction coefficient of a wave behind a barrier from the relative length of the phrase of increase of pressure $\left(\left(\mathrm{C} \tau_{+}\right) / 1_{\mathrm{x}}\right), 1_{\mathrm{x}} / \mathrm{R}=1$.

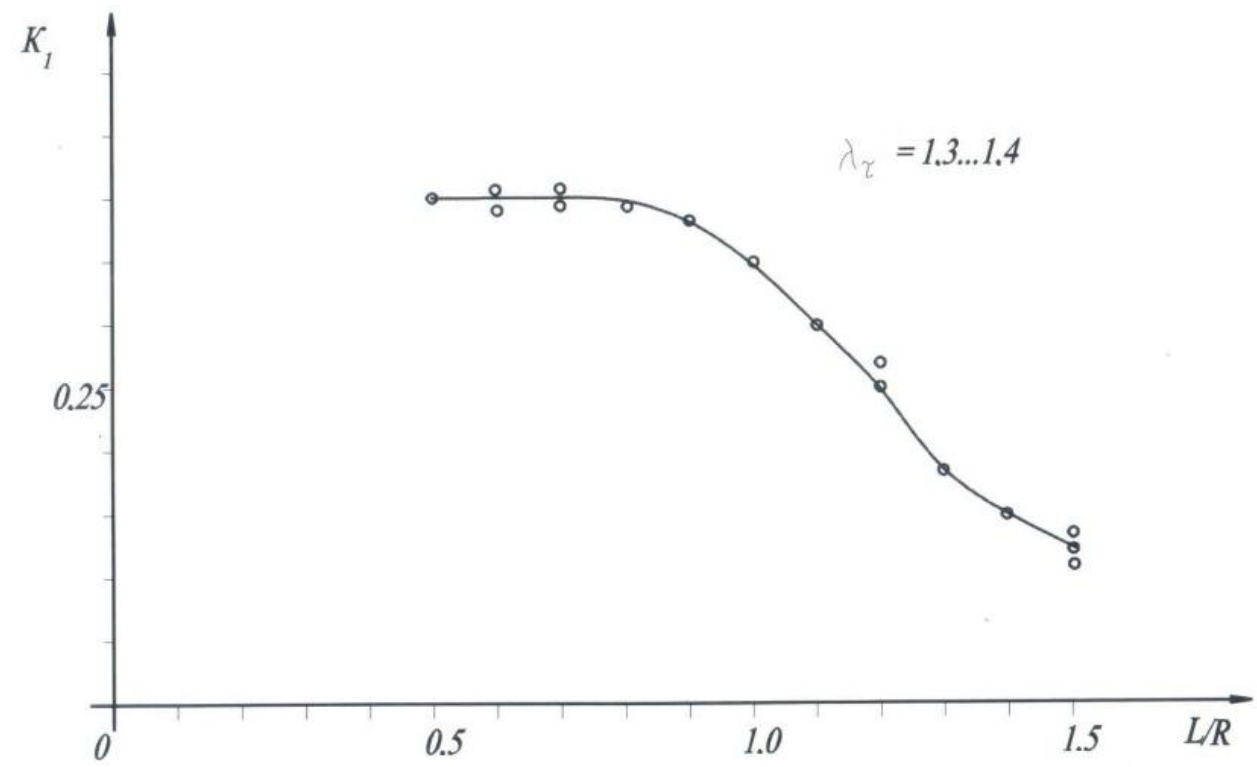

Fig. 4. Weakening of a wave behind a barrier depending on distance to the center of explosion.

The data presented in Figure 3 is obtained under the condition $\mathrm{R}=\mathrm{l}_{\mathrm{x}}$. Obviously, with equal $\bar{\lambda}=C_{0} t_{+} / l_{x}$ and increasing $l_{x} / R$ weakening of the wave parameters for the barrier should be intensified. This is caused by two reasons. The pressure in the wave at the edges of the obstacles is smaller than the free wave at a distance equal to the center of the obstacles. The velocity vector in the blast wave goes away from obstacles. $\mathrm{R}_{1}$ - the distance from the center of the explosion to the sensor behind a barrier.

Table 1 contains information about the attenuation of the wave behind a solid barrier for a wider range of parameters of the waves and obstacles. The coefficients $K_{1}$ and $K_{2}$ is the ratio of the maximum pressure registered by the sensors $\mathrm{D}_{1}$ and $\mathrm{D}_{2}$ to the maximum pressure registered by the sensor $\mathrm{D}_{3}, \mathrm{~K}_{3}$ is the ratio of the maximum pressure registered by the sensor $\mathrm{D}_{4}$ to the pressure sensor $\mathrm{D}_{5}$. 
Table 1. The attenuation of the wave behind a solid barrier.

\begin{tabular}{|c|c|c|c|c|c|c|c|}
\hline № & $\mathbf{R}, \mathbf{M}$ & $\mathbf{R}_{\mathbf{1}} \mathbf{M}$ & $\boldsymbol{\tau}_{+\mathbf{1}}, \mathbf{M c}$ & $\mathbf{I}_{\mathbf{x}}, \mathbf{M}$ & $\mathbf{K}_{\mathbf{1}}$ & $\mathbf{K}_{\mathbf{2}}$ & $\mathbf{K}_{\mathbf{3}}$ \\
\hline 1 & 0.6 & 0.8 & 7.0 & 0.4 & 0.7 & 0.8 & 0.8 \\
\hline 2 & 0.6 & 0.8 & 6.0 & 0.4 & 0.6 & 0.8 & 0.7 \\
\hline 3 & 0.6 & 0.8 & 5.0 & 0.4 & 0.6 & 0.7 & 0.7 \\
\hline 4 & 0.6 & 0.8 & 4.0 & 0.4 & 0.5 & 0.6 & 0.6 \\
\hline 5 & 0.6 & 0.8 & 3.0 & 0.4 & 0.5 & 0.6 & 0.6 \\
\hline 6 & 0.6 & 0.8 & 2.0 & 0.4 & 0.4 & 0.5 & 0.6 \\
\hline 7 & 0.6 & 0.8 & 1.4 & 0.4 & 0.4 & 0.5 & 0.5 \\
\hline 8 & 0.6 & 0.8 & 1.1 & 0.4 & 0.3 & 0.4 & 0.4 \\
\hline 9 & 0.6 & 0.8 & 1.0 & 0.4 & 0.3 & 0.4 & 0.4 \\
\hline 10 & 0.6 & 0.9 & 1.1 & 0.4 & 0.4 & 0.3 & 0.4 \\
\hline 11 & 0.6 & 1.0 & 1.0 & 0.4 & 0.3 & 0.4 & 0.5 \\
\hline 12 & 0.6 & 1.1 & 1.2 & 0.4 & 0.3 & 0.3 & 0.5 \\
\hline 13 & 0.6 & 1.2 & 1.1 & 0.4 & 0.3 & 0.4 & 0.5 \\
\hline 14 & 0.6 & 1.3 & 1.0 & 0.4 & 0.3 & 0.4 & 0.6 \\
\hline 15 & 0.6 & 1.4 & 1.0 & 0.4 & 0.3 & 0.4 & 0.6 \\
\hline 16 & 0.6 & 0.8 & 1.1 & 0.2 & 0.5 & 0.6 & 0.7 \\
\hline 17 & 0.6 & 0.8 & 1.0 & 0.3 & 0.4 & 0.5 & 0.5 \\
\hline 18 & 0.6 & 0.8 & 1.1 & 0.4 & 0.3 & 0.3 & 0.4 \\
\hline 19 & 0.6 & 0.8 & 1.0 & 0.5 & 0.3 & 0.3 & 0.4 \\
\hline 20 & 0.6 & 0.8 & 1.1 & 0.6 & 0.2 & 0.3 & 0.3 \\
\hline 21 & 0.6 & 0.8 & 1.2 & 0.4 & 0.3 & 0.4 & 0.4 \\
\hline 22 & 0.5 & 0.7 & 1.1 & 0.4 & 0.2 & 0.3 & 0.3 \\
\hline 23 & 0.4 & 0.6 & 1.1 & 0.4 & 0.2 & 0.2 & 0.2 \\
\hline & & & & & & & 0.3 \\
\hline
\end{tabular}

From experiments it is seen that the attenuation directly behind the solid obstacle is changed from 0.8 to 0.3 when you decrease the wavelength to the size of the obstacles from 6 to 0.8 (the obstacle was located at a distance of a radius of the cloud of products of combustion from the center of the explosion). The approach of the obstacles to the center of the explosion causes a greater weakening. The results of the experiments it can be concluded that the length of "track" in the wave from the barrier is 3-4 size obstacles, with the length up to a size of the obstacles a pressure reduction is close to the value pressure on the back side.

Moreover, we have made a series of experiments, which used a permeable barrier. We used a single row of the lattice as the barriers in the most of our experiments. The effect of the shape and size of the holes in the barrier were not specifically investigated.

The Table 2 shows the results of experiments with permeable obstacles, combined with the building's model. In all experiments the radius of the cloud of combustion products was $0.2 \mathrm{~m}$, the building's model was located at a distance of $0.5 \mathrm{~m}$ from the center of the 
explosion. The building models and barriers have the same width, half-width of models and the obstacles were greater than their heights. In the table the following notation: $\mathrm{I}$ is the distance between the obstacles and layout, $\mathrm{H}_{\mathrm{ob}}$ - the height of the obstacles, $\mathrm{H}_{\mathrm{m}}$ - the height of the model, $\mathrm{K}_{1}, \mathrm{~K}_{2}, \mathrm{~K}_{3}$ respectively, the ratios of pressure reduction on the front side of the model, the cover and the back side. All ratios were calculated relative to the pressure in the free wave, for the distance of the front side of the model from the center of the explosion. The permeability barrier is 0.4 .

Table 2. The results of experiments with permeable obstacles, combined into a complex model of the building.

\begin{tabular}{|c|c|c|c|c|c|c|}
\hline № & Hob, $\mathbf{M}$ & Hm, $\mathbf{M}$ & $\mathbf{I}, \mathbf{M}$ & $\mathbf{K 1}$ & $\mathbf{K 2}$ & K3 \\
\hline 1 & 0.14 & 0.18 & 0.05 & 0.50 & 0.31 & 0.08 \\
\hline 2 & 0.14 & 0.18 & 0.06 & 0.55 & 0.33 & 0.08 \\
\hline 3 & 0.14 & 0.18 & 0.06 & 0.55 & 0.35 & - \\
\hline 4 & 0.14 & 0.18 & 0.07 & 0.60 & 0.35 & 0.12 \\
\hline 5 & 0.14 & 0.18 & 0.08 & 0.60 & 0.36 & 0.18 \\
\hline 6 & 0.30 & 0.19 & 0.05 & 0.32 & 0.30 & - \\
\hline 7 & 0.30 & 0.19 & 0.06 & 0.31 & 0.28 & 0.05 \\
\hline 8 & 0.30 & 0.19 & 0.06 & 0.30 & 0.25 & 0.05 \\
\hline 9 & 0.30 & 0.19 & 0.07 & 0.28 & 0.24 & - \\
\hline 10 & 0.30 & 0.19 & 0.08 & 0.28 & 0.22 & - \\
\hline 11 & 0.30 & 0.19 & 0.09 & 0.29 & 0.2 & 0.05 \\
\hline
\end{tabular}

The load acting on the front surface of the layout in the experiment is 2-3 times less than the pressure in the transmitted wave, which corresponds to 4-5 times reduction in actual workload based on solid barrier reflection (Table 2). The barriers reduce the load effectively if they are located close to the explosive cloud. It is here that are realized the most high pressure of the explosion and observed the high reflectance of explosive waves.

\section{Results}

Flexible sheet is element and protective barriers.

The connection between the characteristics of the blast wave and the parameters of the flexible sheet, in which it retains its integrity.

This allowed deep plastic deformation. Calculations allow to verify that a sheet of soft (mild) steel can absorb much of the energy of the explosion and serve as the main element in the protective barrier.

The idea of the possible magnitude of plastic deformation prior to failure is shown in Table 3. The data for this table were taken from [19-20].

Table 3. The possible magnitude of plastic deformation.

\begin{tabular}{|c|c|c|c|c|c|c|}
\hline $\begin{array}{c}\text { Material } \\
\text { designation }\end{array}$ & $\begin{array}{c}\text { Tensile } \\
\text { strength } \\
\text { at break, } \\
\text { MPa }\end{array}$ & $\begin{array}{c}\text { The work of } \\
\text { elastic } \\
\text { deformation, } \\
\text { in MPa }\end{array}$ & $\begin{array}{c}\varepsilon_{\mathrm{e}} \text { (elongation } \\
\text { at the elastic } \\
\text { stage })\end{array}$ & $\begin{array}{c}\text { The } \\
\text { Work } \\
\text { at } \\
\text { break } \\
\text { MPa }\end{array}$ & $\begin{array}{c}\boldsymbol{\varepsilon}_{\text {s(static) }} \\
\text { (the } \\
\text { strain } \\
\text { at full } \\
\text { gap) }\end{array}$ & $\begin{array}{c}\boldsymbol{\varepsilon}_{5}(5 \%-\text { is } \\
\text { destroyed, } \\
\text { elongation) }\end{array}$ \\
\hline
\end{tabular}




\begin{tabular}{|c|c|c|c|c|c|c|}
\hline Mild steel & 400 & 0.1 & 0.001 & 110 & 0.4 & 0.2 \\
\hline $\begin{array}{c}\text { Steel } \\
\text { average } \\
\text { hardness }\end{array}$ & 580 & 0.225 & 0.001 & 114 & 0.28 & 0.15 \\
\hline Solid steel & 800 & 0.625 & 0.002 & 36 & 0.05 & 0.03 \\
\hline $\begin{array}{c}\text { Special } \\
\text { alloy steels }\end{array}$ & 1500 & 4.225 & 0.002 & 155 & 0.16 & 0.1 \\
\hline
\end{tabular}

The data shows that mild steel tears up at a very deep plastic deformations. Table 3 introduced the special designation of $\varepsilon_{5}$. This elongation, in which $5 \%$ of the samples get destroyed. Such a designation is accepted in the regulations.

The following conditions must be met:

1. The sheets have to be deformed simultaneously.

2. The deformation of the sheet should be large enough (material saving).

The movement of the sheet is studied numerically. The equation of motion for the variational method with the dynamic hardening with elasto-plastic and rigid-plastic deformation diagram. Sheet is fixed to stationary supports. Dynamic hardening of the material is taken from experiments [20] and is characterized by the average value $\gamma_{d}=1.35$ 1.4. The work of fracture under dynamic loading design of mild steel, is characterized by the multiplier $\gamma_{\mathrm{Ad}}=1.2$. As a result of dynamic strain at failure, $\varepsilon_{\mathrm{d}}=0.87 \varepsilon_{\mathrm{s}}$ For mild steels the rupture strain under static loading $\varepsilon_{\mathrm{s}}=0.4$. Hence dynamic strain fracture $\varepsilon_{\mathrm{d}}==0.35$. Strain $\delta_{5}$ means the destruction of $5 \%$ of the samples of mild steel. Depending on the sample thickness $\varepsilon_{5}=0.2-0.24$, which corresponds to the normative value. If we assume that during dynamic deformation ratio $\varepsilon_{5}=(0.5-0.6) \varepsilon_{\mathrm{s}}$ as with static, it turns out $\varepsilon_{5 \mathrm{~d}}=0.175-0.21$.

The calculated values of $\delta_{5}$, compared to the normative is reduced to 1.1 times, so the calculated value $\varepsilon_{5 \mathrm{~d}}=0.16-0.19$.

Finally, to account for the possibility of uneven deformation of the sheet, accepted a margin of safety $\gamma_{\mathrm{R}}=1.4$, and then the allowable dynamic deformation of the sheet $\varepsilon_{5 \mathrm{~d}}$ $=0.12$. The magnitude of strain $\varepsilon$ is obtained by solving the equations of motion of the sheet [21]. The deflection of the sheet set function $Y_{(x t)}=Y_{0}(t) \sin \frac{\pi x}{L}, 0<\mathrm{x}<\mathrm{L}$, where $\mathrm{Y}_{0}(\mathrm{t})$ is the deflection of the sheet, depending on time. Relative local elongation of the sheet is determined by the expression:

$$
\varepsilon=\frac{1}{2}\left(\frac{d Y}{d X}\right)^{2}=\frac{\pi^{2} Y_{(x, t)}^{2}}{2 L^{2}} \cos ^{2}\left(\frac{\pi x}{L}\right)
$$

The average (length) elongation equal to:

$$
\bar{\varepsilon}=\frac{\pi^{2}}{4} \frac{Y_{0}^{2}(t)}{L^{2}}
$$

The equation of motion of the sheet obtained by the variational method:

$$
\ddot{\bar{Y}}_{0}+\alpha \omega_{0}^{2} \bar{Y}_{0}=\frac{4}{\pi^{3}} \omega_{0}^{2} \beta f(\tau)
$$

Initial condition $\bar{Y}_{0}=0 ; \dot{\bar{Y}}_{0}=0 ; \bar{Y}_{0}=\frac{Y_{0}(t)}{L}$ is the relative deflection of the sheet, L is the length of the sheet. 


$$
\alpha=\left\{\begin{array}{l}
\gamma_{s d} \text { - for plastic stage } \gamma_{s d}=\left[1+\left(\frac{\dot{\varepsilon}_{e}}{40}\right)^{1 / 5}\right] \\
\frac{\pi^{2}}{4} \frac{E}{\sigma_{e}} \bar{Y}^{2}-\text { for the elastic stage, the "e" means "elastic". }
\end{array}\right.
$$

$\omega_{0}=\frac{\pi}{2} \tau_{+} \sqrt{\frac{\sigma_{e}}{\rho}},\left\langle\left\langle\tau_{+} \gg\right.\right.$ is the rise time of the load profile with increasing pressure and fall time for the profile with the shock front;

$$
\beta=\frac{\Delta P_{m} L}{\sigma_{s d} \delta}
$$

$\Delta \mathrm{P}_{\mathrm{m}}$ - is the maximum pressure in the wave in the moment of interaction with the barrier, $\delta$ - is the thickness of the sheet, $\sigma_{\text {sd }}$ is the dynamic yield stress.

At admissible dynamic deformation $\varepsilon_{5}=0.12$ the relative deflection of sheet $\mathrm{Y}_{0} / \mathrm{L}=0.22$.

Follows from the decision that in this case: $\beta=\frac{\Delta P_{m} L}{\sigma_{e} \delta} \approx 2,3$

At $\mathrm{L}=6 \mathrm{~m}, \delta=1 \mathrm{~mm}$, and $\sigma_{\mathrm{e}}=280000 \mathrm{kPa}$ correspond to pressure size in a wave: $\mathrm{P}_{\mathrm{m}}=2,3 * 280000 * 10^{-3} / 6=107 \mathrm{kPa}$.

When the corresponding reinforcement sheet, the magnitude of the blast load on the sheet can be increased by 2-3 times. Enhancement is achieved by increasing the effective thickness of the sheet.

It should be noted that in a significant deflection of the sheet it will pull the attachment point with greater force, which will lead to the weighting structures, which are attached sheets.

In Figure 5 is given the dependence of the maximum strain $\varepsilon_{\max }$ from the dimensionless parameter $\beta$. The numbers (1-5) correspond to waves with a shock front and a trailing portion of the profile of the pressure wave for different frequencies $\omega_{0}$, numbers (6-8) correspond to loads with increasing and decreasing parts of wave profile for different frequencies $\omega_{0}$. 


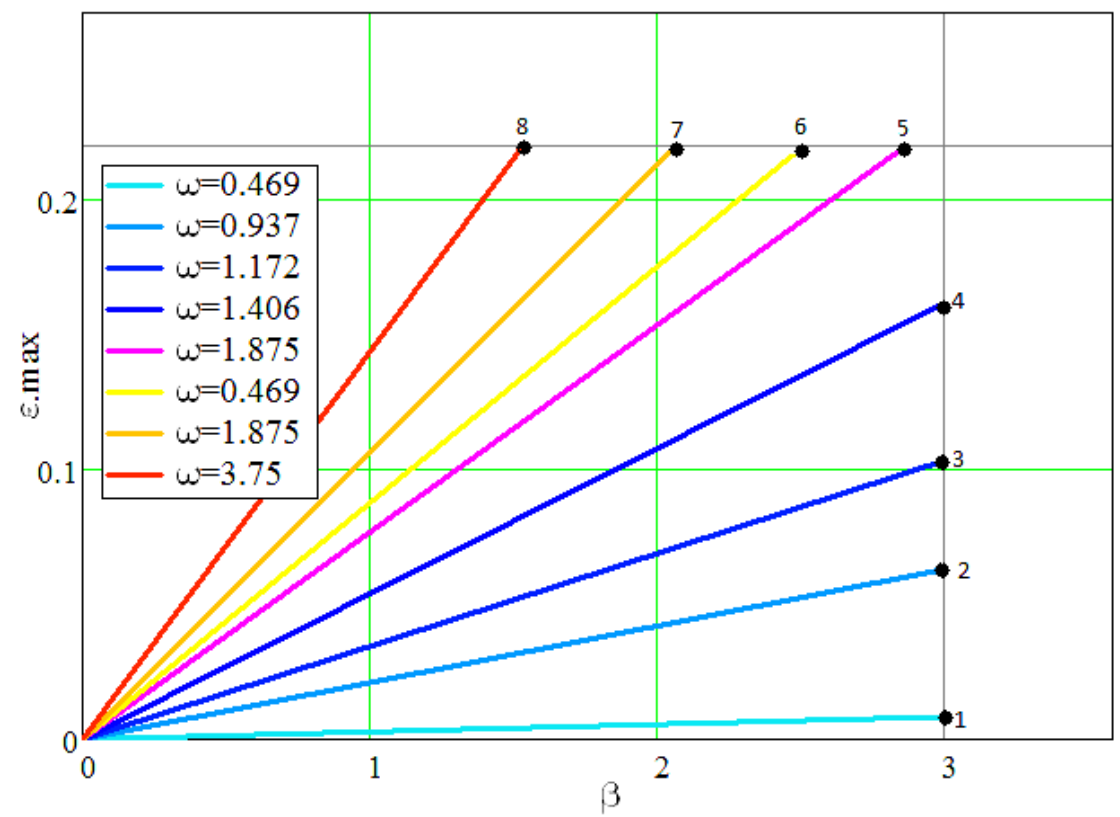

Fig. 5. The dependence of the deformation of a sheet of « $\beta »$ for different frequencies and profiles of the wave.

\section{Discussion}

In [18] experimentally measured the load on the element of a barrier depending on the permeability of the barriers and length of the wave incident on the barrier.

The relative reduction of the maximum pressure at the permeable barrier « $\eta$ » compared to the pressure on a solid obstacle taking into account the reflection was defined as:

$$
\eta=\left(\Delta P_{2}-\Delta P_{\Omega}\right) / \Delta P_{2}
$$

Two cases:

1. The size of the obstacles is less than the wavelength $l_{x} / C_{0} t_{+}<1$. Pressure drop compared to a solid barrier starts with $\Omega>0.2$ and varies from 0.05 to 0.2 when changing $\Omega$ from 0.3 to 0.4 . With a further increase of $\Omega$ value « $\eta$ » does not change.

2. When the size of the obstacles is approximately equal to the wavelength of the pressure drop is more prominent, and « $\eta\rangle$ increases from 0.05 to 0.5 with the increase of $\Omega$ from 0.1 to 0.5 . The load transmitted from the leaves to the supports should be determined taking into account the permeability and pressure action on the back side, the reflection from the object.

\section{Conclusion}

The effect of the external explosion on a building can be reduced with a help of protective barriers being placed in front of the building. Solid barriers, calculated on the carrying capacity for any group of limit condition are heavy, clumsy and therefore expensive. Often their costs are comparable to the costs of the protected object. Perforated barriers with thin metal sheets substantially reduce protection. This effect is due to the tolerance of deep 
plastic deformation of the basic element of the protective barrier, which is proposed as a thin sheet of mild steel.

Deep plastic deformation signals that no more than $5 \%$ of leaves could be collapsed. The relative deformation of the sheet is 0.12 . When such deformation of the sheet is $1 \mathrm{~mm}$ thick and $6 \mathrm{~m}$ long can carry a load corresponding to the excess pressure on the sheet 100 $\mathrm{kPa}$. The sheet can be enhanced by increasing the effective thickness of the sheet several times. If the barrier is permeable, the force on the supports decreases and is a fraction equal to $(1-\Omega)$ of the load perceived by the sheet.

As a result, the punched barriers substantially load of support decrease load of support.

\section{References}

1. R.A. Strehlow, Unconfined vupour explosion-an overview (Symposium in Combustion, Pennsylvania, 1972)

2. B.J. Wiekema, Journal of Hazardous Materials 8, 4 (1984)

3. M.V. Beschastnov, Production explosions. Assessment and prevention (Himiya, Moscow, 1991)

4. V. Marshal, Main dangers of chemical productions (Mir, Moscow, 1989)

5. A.P. Pilrarazio, J.K. Thomas, Q.A. Baker, D.E. Ketchum, Process safety progress 24, 1 (2005)

6. C. Sadee, D.E. Samuels, T.P. O Brien, The characteristics of the explosion of cyclohexane at the Nypro (Flixborough court of inquiry, UK, 1974)

7. The Federal Law 384, Technical Regulations about Safety of Buildings and Constructions (2009)

8. By.D. Bradley, A.K. Lau, M. Lawes, Roy.Soc.Lond.A. 338, 359-387 (1992)

9. C.R. Bauwens, S.B. Dorofeev, Process safety progress, 215 (2015)

10. D.A. Crowl, Understanding explosions center for chemical Process Safity (American institute of chemical Engineers, New York, 2003)

11. C.Y.N. Van den Bosch, Methods for calculation of physical effects (Yellow Book, Ytoffen, 2005)

12. A. Beccantini, I.A. Malczynsk, E. Studer, Comparison of TNT-Equivalency Apprach (Exploision Hazards, Edinburg, 2007)

13. N.A. Ivanov, V.P. Borisovskaya, The Works Tsagi 1834, 26-35 (1977)

14. K.Q. Tong, C.Y. Knight, Srivastava 18, 1298 -1305 (1980)

15. B.E. Gelfand, S.P. Medvedev, A.N. Polenov, S.A. Tsyganov, Arch. Comb. 7, 215-223 (1987)

16. V.A. Gorev, V.N. Fedotov, FGV 6, 79-83 (1986)

17. V.A. Gorev, V.N. Fedotov, Chemical physics 9(12), 1602-1605 (1990)

18. V.A. Gorev, N.V. Tyurin, Fire and explosion safety 2, 21-32 (2005)

19. O.E. Vlasov, Bases of the theory of action of explosion of M. Prod. (Look, Moscow, 1957)

20. P. Saimonds, Dinamika of Inelastic designs (Mir, Moscow, 1982)

21. V.A. Gorev, A.I. Plotnikov, MGSU Bulletin, 4 (2008) 